\title{
Qualité du gamète testiculaire
}

\author{
J.P. DADOUNE
}

Service d'Histologie, Biologie de la Reproduction, Cytogénétique, Hôpital Tenon, Paris

\section{RÉSUMÉ}

Le recueil des spermatozoïdes testiculaires (TESE), suivi de la microinjection dans le cytoplasme ovocytaire (ICSI) a modifié radicalement la prise en charge des patients présentant une azoospermie sécrétoire. Cependant, les anomalies du processus spermatogénétique conduisent à s'interroger sur la qualité des gamètes recueillis au cours de la biopsie testiculaire quand on sait que le spermatozoïde est l'aboutissement d'une évolution séquentielle et programmée. Cette revue a pour objet :

1. De rappeler brièvement les modalités de synthèse des protéines constitutives du spermatozoïde ;

2. De préciser les caractéristiques des spermatozoïdes prélevés dans le testicule ;

3. D'évaluer l'incidence des anomalies nucléaires sur les résultats cliniques de l'ICSI.

Mots clés : spermatozoide, ICSI, apoptose, anomalies nucléaires.

\section{INTRODUCTION}

Le recueil de spermatozoides testiculaires (TESE) suivi de la micro-injection dans le cytoplasme ovocytaire (ICSI) a modifié radicalement le traitement des hommes présentant une azoospermie en relation avec un déficit de la spermatogenèse [45]. Le taux de spermatozoïdes collectés par biopsie testiculaire reste relativement élevé aussi bien au cours de l'aplasie germinale complète (de l'ordre de 19 $\%$ ) et de l'arrêt complet de la maturation spermatogénétique (de l'ordre de $48 \%$ ) que de la sclérose tubulaire (de l'ordre de $40 \%$ ) [42]. Cependant, une étude comparative effectuée après ICSI avec des spermatozoïdes éjaculés, épididymaires et testiculaires a montré une réduction de moitié du pourcentage de nouveaux nés vivants par embryons transférés, ainsi qu'un taux de fausses couches deux à trois fois plus élevé après usage de spermatozoïdes testiculaires [10].

Cette limitation sensible du potentiel de développement des embryons engendrés avec des spermatozoïdes testiculaires conduit à s'interroger sur la qualité des gamètes obtenus à partir de testicules où le processus spermatogénétique est fortement perturbé. Le spermatozoïde est, en effet, l'aboutissement d'une évolution séquentielle et programmée qui se poursuit tout au long de la spermatogenèse. On peut donc supposer que non seulement les anomalies de la spermiogenèse, mais aussi celles de la lignée germinale survenues en amont retentissent sur l'état des spermatozoïdes.

Correspondance : Jean-Pierre Dadoune, Service d'Histologie, Biologie de la Reproduction, Cytogénétique, Hôpital Tenon, 4 rue de la Chine, 75970 PARIS cedex 20.

Communication au XVIIème Congrès de la SALF, 7-9 décembre 2000, Bordeaux. 
Cette revue a pour objet :

1) de rappeler brièvement les modalités de synthèse des protéines constitutives du spermatozoïde,

2) de préciser les caractéristiques des spermatozoïdes prélevés dans le testicule et

3) d'évaluer l'incidence des anomalies nucléaires sur les résultats cliniques de l'ICSI.

\section{SYNTHÈSE SÉQUENTIELLE DES PROTÉINES NUCLÉAIRES ET CYTO- PLASMIQUES DU SPERMATOZOÏDE}

Les modalités de synthèse des ARN messagers sont particulières à la lignée germinale mâle. Les ARNm peuvent être synthétisés de manière continue dans les trois familles de cellules germinales, spermatogonies, spermatocytes et spermatides. Certains ARNm méiotiques avec une demi-vie inhabituellement longue sont transcrits et stockés dans les spermatocytes I pour être traduits beaucoup plus tard dans les jeunes spermatides. A l'opposé, d'autres ARNm ne sont transcrits que dans les spermatides (expression du génome haploïde). De la sorte, les protéines constitutives des spermatozoïdes sont le produit d'une activité transcriptionnelle et traductionnelle à la fois mitotique, méiotique et post-méiotique [12]. Une étude ancienne, mais fondamentale, de la séquence d'apparition des protéines dans les spermatozoïdes chez la souris, a établi que les protéines solubles en provenance de la membrane plasmique, de l'acrosome, de l'axonème, de la matrice et des crêtes mitochondriales sont élaborées principalement au cours de la méiose. Au contraire, la synthèse des composants insolubles de la tête et de la queue s'effectue durant la spermiogenèse [26].

Les remaniements nucléaires, une étape essentielle de la différenciation des spermatozoïdes, surviennent à mi-course de la spermiogenèse. Chez l'homme, les histones de type somatique et leurs variants testicule-spécifiques sont remplacées par deux protéines de transition (TP1 et TP2) qui, à leur tour, sont déplacées par les protamines (familles P1 et $\mathrm{P2}$ ), dès la fin de la phase d'élongation des spermatides [17, 30]. L'interaction inter et intra-protamines due à la formation des ponts disulfure entre les résidus cystéine favorise la compaction de l'ADN. Les séquences des protéines de transition et des protamines sont connues. Il en est de même pour les gènes correspondants dont l'activité est strictement limitée aux spermatides (références in [7]). Les modalités de la régulation transcriptionnelle et traductionnelle de leur expression commencent à être élucidées $[13,8,37]$. C'est le cas, en particulier, d'une protéine de liaison à l'ARN, la TRBP (TAR RNA binding protein), homologue de la Prbp de souris (Prm-1 RNA binding protein) dont l'existence a été récemment démontrée dans les spermatides humaines en élongation et qui intervient vraisemblablement dans le contrôle traductionnel des protamines [36].

La qualité nucléaire, en d'autres termes l'intégrité du complexe ADN-protamines, est, à l'évidence, un facteur déterminant de la fécondation de l'ovocyte et du développement embryonnaire, après ICSI. Les anomalies de condensation de la chromatine se constituent au cours de la spermiogenèse. En revanche, la fragmentation de l'ADN peut éventuellement être l'expression d'un processus apoptotique affectant l'ensemble de la lignée germinale.

\section{CARACTÉRISTIQUES DES SPERMATOZOÏDES TESTICULAIRES}

L'examen à l'état frais des spermatides matures et des spermatozoïdes recueillis par biopsie testiculaire est certainement informatif quant aux dimensions et à la forme générale des cellules. En effet, l'aspect des cellules vivantes paraît, en tous points semblable à celui observé sur les préparations histologiques comme l'a montré une étude morphologique réalisée conjointement sur des cellules germinales en culture et sur les coupes de tubes séminifêres en provenance des mêmes prélèvements [15].

L'évaluation des paramètres usuels des spermatozoïdes testiculaires collectés au cours de l'azoospermie sécrétoire indique que les cellules conservent une vitalité élevée (environ 86 $\%$ de formes vivantes après coloration vitale et $60 \%$ avec le test de gonflement hypoosmotique - HOS test ) en dépit d'une diminution importante du nombre et de la mobilité (environ $5 \%$ 
de formes mobiles) [2]. Il faut souligner que les tests de vitalité permettent d'identifier beaucoup plus de formes viables que ne le suggère l'examen de la mobilité (tableau 1).

Néanmoins, la mobilité reste un critère important du choix du gamète. Après injection de spermatozoïdes testiculaires mobiles, le taux de fécondation est significativement plus élevé qu'après injection de spermatozoïdes non mobiles [25] (tableau 2).

En revanche, dans les conditions du recueil, on ne dispose d'aucune information sur la condensation de la chromatine et l'état de l'ADN. En dépit d'une forme de la tête normale ou subnormale, les spermatozoïdes peuvent toutefois présenter des anomalies variables de condensation de la chromatine qui ne sont décelables qu'en microscopie électronique (figure 1).

\section{INCIDENCES DES ANOMALIES NUCLÉAIRES SUR LES RÉSULTATS DE L'ICSI}

Diverses méthodes ont été employées pour apprécier la qualité de la chromatine des spermatozoïdes. Parmi celles-ci, la coloration par la chromomycine A3 (CRM 3), un fluorochrome spécifique des séquences guanine-cytosine de l'ADN, permet de révéler un défaut de protamination [4]. La nick-translation in situ sans traitement préalable par une endonucléase met en évidence la fragmentation de l'ADN [5]. Les résultats fournis par cette méthode sont d'ailleurs identiques à ceux obtenus avec la méthode TUNEL (Terminal deoxynucleotidyl transferase - mediated dUTP-Nick-EndLabeling) utilisée pour détecter la dégradation de l'ADN dans les cellules somatiques apoptiques [21].

\section{a) Anomalies de l'interaction ADN-prota- mines}

L'étude de la chromatine avec la chromomycine A3 et la nick translation in situ chez les patients candidats à l'ICSI a montré une augmentation significative des anomalies de condensation de la chromatine [35], le défaut de protamination étant généralement associé à la persistance de nicks endogènes d'ADN [20]. Bien qu'elles n'aient pas d'incidence sur les taux de fécondation proprement dits, ces ano- malies nucléaires restent un facteur limitant de la fécondation [35]. En effet, chez les patients présentant un pourcentage élevé d'anomalies de la chromatine ( $30 \%$ de spermatozoïdes CRM3-positifs, > $10 \%$ de spermatozoïdes avec $\mathrm{ADN}$ fragmenté), plus du double des ovocytes non fécondés contiennent des spermatozoïdes à tête non décondensée [34]. En outre, quand on effectue la culture sur cellules Vero d'embryons surnuméraires engendrés soit par ICSI, soit par FIV avec des spermatozoïdes porteurs d'anomalies nucléaires, le pourcentage de blastocytes est significativement plus bas après ICSI $(26,8 \%$ contre $47,3 \%$ ), suggérant l'intervention d'une sélection des embryons durant la période préimplantatoire [35].

\section{b) Altération de l'ADN}

En général, le taux de fécondation après ICSI n'excède pas $65 \%$, alors que le spermatozoïde est introduit directement dans l'ovocyte mature $[27,28,40]$. Ce taux de fécondation inférieur à ce qui est attendu pourrait s'expliquer par la présence d'anomalies de l'ADN dans les spermatozoïdes chez des patients par ailleurs infertiles.

Les spermatozoïdes éjaculés dont la fragmentation de l'ADN est détectée par la méthode TUNEL ont des caractéristiques ultrastructurales analogues à celles des cellules somatiques apoptotiques : chromatine partiellement fragmentée et condensée à la périphérie, rupture ou disparition de la membrane nucléaire, ballonisation des mitochondries. Le pourcentage de spermatozoïdes à $\mathrm{ADN}$ fragmenté dans l'éjaculat peut varier de 1 à $50 \%$ suivant les pathologies de l'appareil génital [1]. Il est corrélé négativement au pourcentage de formes mobiles chez les hommes infertiles [9]. De plus, les taux de fécondation et de clivage embryonnaire après fécondation in vitro [39] ou ICSI [19] sont d'autant plus bas que le pourcentage de spermatozoïdes avec ADN fragmenté est plus élevé.

Un travail récent réalisé à la fois chez l'homme et des souris transgéniques montre que le risque de fragmentation de l'ADN est accru dans les cellules germinales mâles quand il y a altération de la spermatogenèse [16]. En effet, chez les mutants homozygotes de souris (pro- 


\begin{tabular}{lccc}
\hline Origine & Mobilité $(\%)$ & vitalité $(\%)$ & HOS test (\%) \\
\hline Tegticule $(\mathbf{a})(\mathbf{n}=5)$ & $5+3.6$ & $86+5$ & $60+12$ \\
Epididyme $(\mathrm{b})(\mathrm{n}=8)$ & $22+18$ & $57+20$ & $40+14$ \\
Canal déférent $(\mathrm{c})(\mathbf{n}=\mathbf{5})$ & $71+16$ & $\mathbf{9 1 + 6}$ & $80+11$ \\
\hline
\end{tabular}
(a) Azoospermie sécrétoire (TESE)
(b) Azoospermie obstructive (MESA)
(c) Avant vasectomie (modifié d'après [2])

Tableau 2 : Résultats de l'ICSI en fonction de la mobilité des spermatozoüdes testiculaires

\begin{tabular}{lccc}
\hline & $\begin{array}{c}\text { Groupe 1 } \\
\text { sptz mobiles }\end{array}$ & $\begin{array}{c}\text { Groupe 2 } \\
\text { sptz immobiles }\end{array}$ & Total \\
\hline Nombre de cycles & 97 & 8 & 129 \\
Nombre d'ovocytes injectés & 810 & 89 & 1042 \\
2 PN (\%) & $\approx 65^{*}$ & $\simeq 21$ & $\approx 60$ \\
$\begin{array}{l}\text { Embryons transférés } \\
\text { ou congelés (\%) }\end{array}$ & $\simeq 69$ & $\simeq 84$ & $\simeq 71$ \\
\hline
\end{tabular}

* Significativement différent (modifié d'après [25])

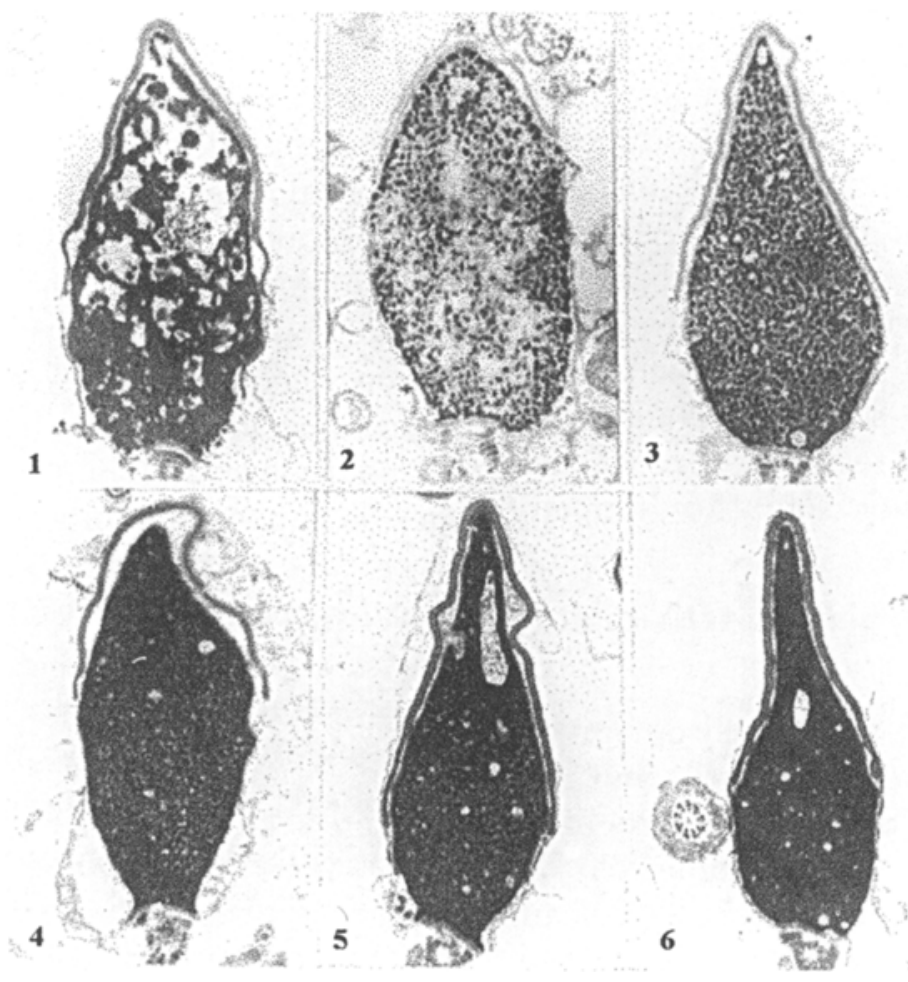

Figure 1: Ultrastructure de têtes de spermatozoüdes humains de forme normale ou subnormale présentant des degrés variables d'anomalies de condensation de la chromatine ( $x$ 6000). 
téine phosphase $1 \mathrm{c} \gamma-/$-) dont la spermiogenèse est bloquée, la fragmentation de l'ADN est significativement augmentée dans les spermatozoïdes testiculaires $(28,7 \%$ contre $4,1 \%$ dans la souche sauvage) et les spermatides rondes $(27,4 \%$ contre $3,9 \%$ dans la souche sauvage) (tableau 3). Chez l'homme un taux élevé de fragmentation de l'ADN est également observé dans les spermatides rondes au cours de l'azoospermie sécrétoire et dans ce cas, le dommage de l'ADN compromet le développement embryonnaire. Dans la série étudiée, chez six patients présentant un pourcentage élevé de spermatides rondes avec ADN fragmenté, aucune grossesse n'a été obtenue avec les spermatozoïdes testiculaires, alors que chez un patient dépourvu de spermatozoïdes, une grossesse est survenue avec des spermatides rondes où la fragmentation de l'ADN était deux fois moindre.

\section{c) Relation entre la fragmentation de l'ADN et l'apoptose}

A ce jour, les facteurs responsables de la fragmentation de l'ADN dans les spermatozoïdes ne sont pas clairement élucidés. La présence d'ADN fragmenté pourrait être en relation avec le processus de compaction de la chromatine au cours de la spermiogenèse. Il a été montré, en effet, que des nicks endogènes d'ADN sont normalement présents dans le noyau des spermatides en élongation chez les rongeurs $[22,23,33]$. La formation de nicks endogènes de l'ADN bicaténaire aurait pour effet de favoriser les interactions des protamines avec l'ADN. Cependant, ces fragments disparaissent dès que la maturation nucléaire est achevée. Les topoisomérases, considérées comme les enzymes responsables de la fragmentation de l'ADN sont présentes au cours de la spermatogenèse $[6,24]$. Des anomalies de la fonction ligase seraient à l'origine de la persistance des nicks endogènes et de l'altération de la structure de la chromatine dans les spermatozoïdes des hommes hypofertiles [4, 20, 32, 34].

La fragmentation de l'ADN dans les spermatozoïdes matures pourrait être aussi causée par un excès de radicaux libres dans le plasma séminal. En effet, le stress oxydatif est un facteur reconnu de l'altération de l'ADN dans le gamète mâle $[14,43,44]$. Une relation directe a été établie entre la fragmentation de l'ADN, la redistribution de la phosphatidylsérine à la surface de la membrane plasmique et la production de radicaux libres endogènes chez les hommes infertiles [3].

En troisième lieu, le dommage de l'ADN dans les spermatozoïdes serait la conséquence de l'apoptose, ou mort cellulaire programmée, en tous points semblable à celle qui affecte les cellules somatiques $[11,39]$. Dans ce contexte, l'apoptose peut conduire à l'élimination sélective des cellules germinales endommagées comme le suggère l'incidence élevée de l'apoptose dans les spermatocytes et les spermatides chez les hommes inféconds [41]. Au cours des anomalies de la spermatogenèse, le taux des cellules apoptotiques est d'autant plus élevé que l'arrêt de la spermatogenèse est plus précoce [18]. On comprend dès lors l'intérêt d'apprécier en amont de la spermiogenèse l'équilibre entre l'apoptose et la prolifération cellulaire, les deux processus antagonistes qui assurent l'homéostasie de chacune des familles cellulaires de la lignée germinale [31] (figure 2). Ainsi, la biopsie testiculaire à visée pronostique, pratiquée avant l'ICSI, retrouve son utilité pour évaluer les proportions respectives de cellules germinales en apoptose ou en prolifération. On dispose en effet maintenant de marqueurs immunocytochimiques appropriés tels que l'anticorps dirigé contre la protéine Fas qui s'accumule dans les spermatocytes et les spermatides apoptotiques [29] et les anticorps dirigés contre les protéines $\mathrm{Ki}-67$ et PCNA (Proliferating Cell Nuclear Antigen) qui s'expriment dans le noyau des cellules germinales en renouvellement |38].

\section{CONCLUSION}

Il est difficile encore actuellement de déterminer avec précision la part respective de l'apoptose et de l'équipement défectueux en protamines dans les anomalies de condensation de la chromatine dans les spermatides matures et les spermatozoïdes. Quelle que soit leur nature, ces anomalies retentissent, semble-t-il, d'avantage sur le développement embryonnaire que sur la fécondation proprement dite. Or, parmi les paramètres spermatiques, la qualité nucléaire est le critère le plus difficile à appré- 
Tableau 3 : Fragmentation de l'ADN dans les cellules germinales haploïdes chez des mutants de souris présentant un blocage de la spermiogenèse (Mutation du gène Pp1cy)

\begin{tabular}{|c|c|c|c|}
\hline & $+/+$ & $+/-$ & $-/-$ \\
\hline \% sptz épididymaires & $\begin{array}{c}1,1+0,6^{*} \\
(\mathbf{n}=6)\end{array}$ & $\begin{array}{c}1,6+0,5 \\
(\mathrm{n}=6)\end{array}$ & $\begin{array}{c}34,3+9,9^{*} \\
(n=9)\end{array}$ \\
\hline \% sptides rondes épididymaires & 0 & 0 & $\begin{array}{c}39,8+4,1 \\
(\mathrm{n}=9)\end{array}$ \\
\hline \% sptides testiculsires & $\begin{array}{c}4,1+0,7^{*} \\
(n=7)\end{array}$ & $\begin{array}{l}4,2+2 \\
(n=7)\end{array}$ & $\begin{array}{c}28,7+8,1^{*} \\
(\mathbf{n}=9)\end{array}$ \\
\hline$\%$ sptides rondes testiculaires & $\begin{array}{c}3,9+1,5^{*} \\
(\mathrm{n}=7)\end{array}$ & $\begin{array}{c}5,4+3,8 \\
(\mathrm{n}=7)\end{array}$ & $\begin{array}{c}27,4+6,8^{*} \\
(\mathrm{n}=9)\end{array}$ \\
\hline
\end{tabular}

* Significatif (modifié d'après [16])

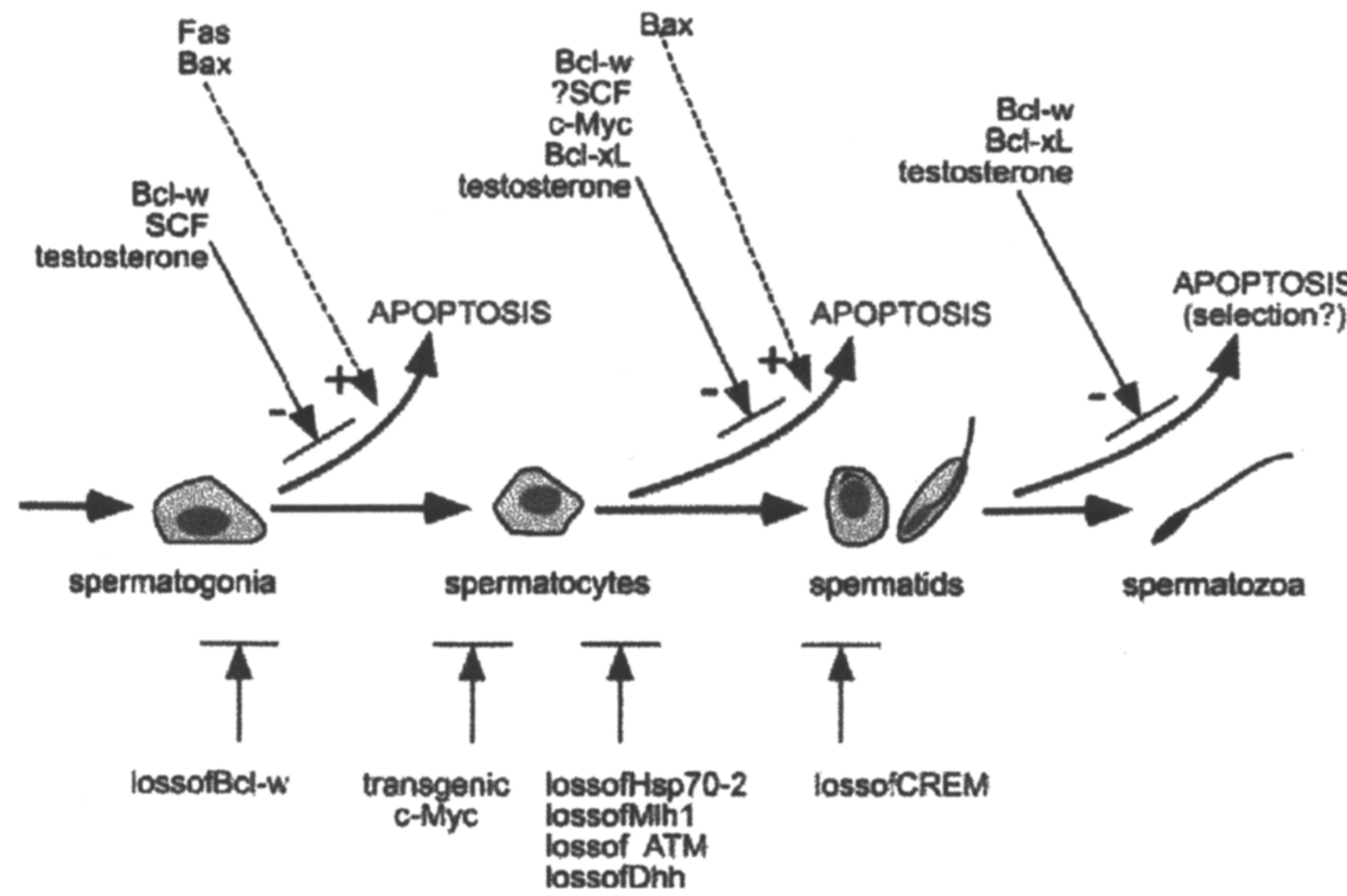

Figure 2: Durant la spermatogenèse, l'apoptose des cellules germinales maintient un nombre approprié de chaque type de cellules germinales et aussi élimine sélectivement les cellules altérées. Les signaux qui régulent l'apoptose sont indiqués dans la partie supérieure du schéma. Les effets des mutations des gènes codant pour les régulateurs de l'apoptose sont représentés dans la partie inférieure [31]. 
cier. Dans ce contexte, il paraît raisonnable de formuler trois recommandations avant l'usage de spermatozoïdes testiculaires : 1) Déterminer la cause précise de l'azoospermie sécrétoire ; 2) Introduire une analyse histologique de la biopsie testiculaire à visée prédictive ; 3 ) Pratiquer systématiquement un conseil génétique.

\section{RÉFÉRENCES}

1. BACCETTI B., COLLODEL G., PIOMBONI P. : Apoptosis in human ejaculated sperm cells (notulae seminologicae 9). J. Submicrosc. Cytol. Pathol., 1996, $28: 587-596$.

2. BACHTELL N.E., CONAGHAN J., TUREK P.J. : The relative viability of human spermatozoa from the vas deferens, epididymis and testis before and after cryopreservation. Hum. Reprod., 1999, 14 : 3048-3051.

3. BARROSO G., MORSHEDI M., OEHNINGER S. : Analysis of DNA fragmentation, plasma membrane translocation of phosphatidylserine and oxidative stress in human spermatozoa. Hum. Reprod., 2000, $15: 1338-1344$.

4. BLANCHI P.G., MANICARDI G.C., BIZZARO D., BIANCHI U., SAKKAS D. : Effect of deoxyribonucleic acid protamination on fluorochrome staining and in situ nick-translation of murine and human mature spermatozoa. Biol. Reprod., 1993, 49 : 10831088.

5. BIZZARO D., MANICARDI G.C., BIANCHI P.G., BIANCHI U., MARIETHOZ E., SAKKAS D. : In-situ competition between protamine and fluorochromes for sperm DNA. Mol. Hum. Reprod., 1998, 4 : 127 132.

6. CHEN J.L., LONGO F.J. : Expression and localization of DNA topoisomerase II during rat spermatogenesis. Mol. Reprod. Dev., 1996, 45 : 61-71.

7. DADOUNE J.P. : The nuclear status of human sperm cells. Micron, 1995, $26: 323-345$.

8. EDDY E.M. : Regulation of gene expression during spermatogenesis. Semin. Cell. Dev. Biol., 1998, 9 : 451-457.

9. GANDINI L., LOMBARDO F., PAOLI D., CAPONECCHIA L., DONDERO F. : Apoptosis in ejaculated human spermatozoa. Int. J. Androl., 1998, 21 (suppl) $: 16$.

10. GHAZZAWI I.M., SARRAF M.G., TAHER M.R., KHALIFA F.A. : Comparison of the fertilizing capability of spermatozoa from ejaculates, epididymal aspirates and testicular biopsies using intracytoplasmic sperm injection. Hum. Reprod., 1998, 13 : 348-352.

11. GORCZYCA W., TRAGANOS F., JESIONOWSKA H., DARZYNKIEWICZ Z. : Presence of DNA strand breaks and increased sensitivity of DNA in situ to denaturation in abnormal human sperm cells: analogy to apoptosis of somatic cells. Exp. Cell. Res., 1993, $207: 202-205$.

12. HECHT N.B. : The making of a spermatozoon: a molecular perspective. Dev. Genet., 1995, 16 : 95-103.

13. HECHT N.B. : Molecular mechanisms of male germ cell differentiation. Bioessays, 1998, 20 : 555-561.

14. HUGHES C.M., MCKELVEY-MARTIN V.J., LEWIS S.E. : Human sperm DNA integrity assessed by the Comet and ELISA assays. Mutagenesis, 1999, 14 : 71-75.

15. JOHNSON L., NEAVES W.B., BARNARD J.J., KEILLOR G.E., BROWN S.W., YANAGIMACHI R. : A comparative morphological study of human germ cells in vitro or in situ within seminiferous tubules. Biol. Reprod., 1999, 61 : 927-934.

16. JURISICOVA A., LOPES S., MERIANO J., OPPEDISANO L., CASPER R.F., VARMUZA S. : INA damage in round spermatids of mice with a targeted disruption of the Pplcgamma gene and in testicular biopsies of patients with non-obstructive azoospermia. Mol. Hum. Reprod., 1999, 5 : 323-330.

17. LE LANNIC G., ARKHIS A., VENDRELY E., CHEVAILLIER P., DADOUNE J.P. : Production, characterization, and immunocytochemical applications of monoclonal antibodies to human sperm protamines. Mol. Reprod. Dev., 1993, 36 : 106-112.

18. LIN W.W., LAMB D.J., WHEELER T.M., LIPSHULTZ L.I., KIM E.D. : In situ end-labeling of human testicular tissue demonstrates increased apoptosis in conditions of abnormal spermatogenesis. Fertil. Steril., 1997, $68: 1065-1069$.

19. LOPES S., SUN J.G., JURISICOVA A., MERIANO J., CASPER R.F. : Sperm deoxyribonucleic acid fragmentation is increased in poor-quality semen samples and correlates with failed fertilization in intracytoplasmic sperm injection. Fertil. Steril., 1998, $69: 528-532$.

20. MANICARDI G.C., BIANCHI P.G., PANTANO s., et al. : Presence of endogenous nicks in DNA of ejaculated human spermatozoa and its relationship to chromomycin A3 accessibility. Biol. Reprod., 1995, 52 : 864-867.

21. MANICARDI G.C., TOMBACCO A., BIZZARO D., BIANCHI U., BIANCHI P.G., SAKKAS D. : DNA strand breaks in ejaculated human spermatozoa : comparison of susceptibility to the nick translation and terminal transferase assays. Histochem J., 1998, $30: 33-39$

22. MCPHERSON S., LONGO F.J. : Chromatin structure-function alterations during mammalian spermatogenesis : DNA nicking and repair in elongating spermatids. Eur. J. Histochem., 1993, 37 : 109-128.

23. MCPHERSON S.M., LONGO F.J. : Nicking of rat spermatid and spermatozoa DNA : possible involvement of DNA topoisomerase II. Dev. Biol., 1993, 158: 122-130. 
24. MORSE-GAUDIO M., RISLEY M.S. : Topoisomerase II expression and VM-26 induction of DNA breaks during spermatogenesis in Xenopus laevis. J. Cell. Sci., 1994, 107 : 2887-2898.

25. NAGY Z.P., VERHEYEN G., TOURNAYE H., VAN STEIRTEGHEM A.C. : Special applications of intracytoplasmic sperm injection: the influence of sperm count, motility, morphology, source and sperm antibody on the outcome of ICSI. Hum. Reprod. 1998, 13 (Suppl. 1) : 143-154.

26. O'BRIEN D.A., BELLVE A.R. : Protein constituents of the mouse spermatozoon. II. Temporal synthesis during spermatogenesis. Dev. Biol., 1980, 75 : 405418.

27. PALERMO G.D., COHEN J., ALIKANI M., ADLER A., ROSENWAKS Z : Development and implementation of intracytoplasmic sperm injection (ICSI). Reprod. Fertil. Dev., 1995, 7 : 211-217.

28. PAYNE D., MA'TTHEWS C.D. : Intracytoplasmic sperm injection-clinical results from the reproductive medicine unit, Adelaide. Reprod. Fertil. Dev., 1995, $7: 219-227$.

29. PENTIKAINEN V., ERKKILA K., DUNKEL L. : Fas regulates germ cell apoptosis in the human testis in vitro. Am. J. Physio., 1999, 276 : E 310-316.

30. PRIGENT Y., MULLER S., DADOUNE J.P. : Immunoelectron microscopical distribution of histones $\mathrm{H} 2 \mathrm{~B}$ and $\mathrm{H3}$ and protamines during human spermiogenesis. Mol. Hum. Reprod., 1996, 2 : 929935.

31. PRINT C.G., LOVELAND K.L. : Germ cell suicide: new insights into apoptosis during spermatogenesis. Bioessays, 2000, 22 : 423-430.

32. SAILER B.L., JOST L.K., EVENSON D.P. : Mammalian sperm DNA susceptibility to in situ denaturation associated with the presence of DNA strand breaks as measured by the terminal deoxynucleotidyl transferase assay. J. Androl., 1995, $16: 80$ 87.

33. SAKKAS D., MANICARDI G., BIANCHI P.G., BIZZARO D., BLANCHI U. : Relationship between the presence of endogenous nicks and sperm chromatin packaging in maturing and fertilizing mouse spermatozoa. Biol. Reprod., 1995, 52 : 1149-1155.

34. SAKKAS D., URNER F., BIANCHI P.G., et al. : Sperm chromatin anomalies can influence decondensation after intracytoplasmic sperm injection. Hum. Reprod., 1996, 11 : 837-843.

35. SAKKAS D., URNER F., BIZZARO D., et al. : Sperm nuclear DNA damage and altered chromatin structure: effect on fertilization and embryo development. Hum. Reprod., 1998, 13 (Suppl. 4) : 11-19.

36. SIFFROI J.P., PAWIAK A., AI.FONSI M.F., TROALEN F., GUELlAEN G., DADOUNE J.P. : Expression of the TAR RNA binding protein in human testis. Mol. Hum. Reprod., 2001, 7 : 219-225.

37. STEGER K. : Transcriptional and translational regulation of gene expression in haploid spermatids. Anat. Embryol., (Berl) 1999, 199 : 471-487.

38. STEGER K., ALEITHE I., BEHRE H., BLRGMANN M. : The proliferation of spermatogonia in normal and pathological human seminiferous epithelium: an immunohistochemical study using monoclonal antibodies against $\mathrm{Ki}-67$ protein and proliferating cell nuclear antigen. Mol. Hum. Reprod., 1998, 4 : 227233.

39. SUN J.G., JURISICOVA A., CASPER R.F. : Detection of deoxyribonucleic acid fragmentation in human sperm: correlation with fertilization in vitro. Biol. Reprod., 1997, 56 : 602-607.

40. SVALANDER P., FORSBERG A.S., JAKOBSSON A.H., WIKLAND M. : Factors of importance for the establishment of a successful program of intracytoplasmic sperm injection treatment for male infertility. Fertil. Steril., 1995, $63: 828-837$.

41. TESARIK J., GRECO E., COHEN-BACRIE P., MENDOZA C. : Germ cell apoptosis in men with complete and incomplete spermiogenesis failure. Mol. Hum. Reprod., 1998, $4: 757-762$.

42. TOURNAYE H., VERHEYEN G., NAGY P., et al. : Are there any predictive factors for successful testicular sperm recovery in azoospermic patients? Hum. Reprod., 1997, 12 : 80-86.

43. TWIGG J., FULTON N., GOMEZ E., IRVINE D.S., AITKEN R.J. : Analysis of the impact of intracellular reactive oxygen species generation on the structural and functional integrity of human spermatozoa: lipid peroxidation, DNA fragmentation and effectiveness of antioxidants. Hum. Reprod., 1998, 13 : 1429-1436.

44.TWIGG J., IRVINE D.S., HOUSTON P., FULTON N., MICHAEL L., AITKEN R.J. : Iatrogenic DNA damage induced in human spermatozoa during sperm preparation: protective significance of seminal plasma. Mol. Hum. Reprod., 1998, 4 : 439-445.

45. VAN STEIRTEGHEM A., NAGY P., JORIS H. et al. : The development of intracytoplasmic sperm injection. Hum. Reprod., 1996, 11 Suppl 1 : 59-72 ; discussion 81-85. 


\section{ABSTRACT \\ Testicular gamete quality}

\section{J.P. DADOUNE}

The combination of testicular sperm extraction (TESE) and ICSI has constituted a breakthrough in the treatment of men with nonobstructive azoospermia. However, impaired spermatogenesis raises the question of the quality of testicular spermatozoa obtained by testicular biopsy, when considering the male gamete as the product of a sequential and programmed spermatogenic process. The aim of this paper is: 1) To briefly recall the successive events of sperm protein synthesis; 2) To define the characteristics of sperm cells retrieved from the testis and 3) To evaluate the effect of nuclear anomalies on the outcome of ICSI.

Key-words: Sperm, ICSI, apoptosis, nuclear abnormalities. 